\section{Psoriasis does not affect the 5-year outcomes of patients with inflammatory polyarthritis}

Patients with psoriasis have an increased risk of developing inflammatory polyarthritis, but it is generally accepted that psoriasis plus inflammatory polyarthritis does not necessarily result in psoriatic arthritis (PsA). The frequency of severe PsA is unclear; some reports suggest that PsA is usually mild, whereas others propose that disease severity progressively worsens over time. Morgan and colleagues studied whether patients with psoriasis plus inflammatory polyarthritis had different 5-year outcomes from patients with inflammatory polyarthritis only, without attempting to classify patients as having PsA.

The study included 79 patients with psoriasis plus inflammatory polyarthritis and 755 patients with inflammatory polyarthritis only; all patients had been recruited to the Norfolk Arthritis Register between 1990 and 1994. Participants were followed-up annually for 5 years.

At baseline, patients with psoriasis were more likely to be male, younger at arthritis onset, and less likely to be rheumatoid factor (RF)-positive, compared with patients without psoriasis. At 5 years, the percentages of patients in remission and of those who satisfied the 1987 ACR criteria for rheumatoid arthritis did not differ between groups. Patients with psoriasis remained less likely to be RF-positive compared with patients without psoriasis (adjusted odds ratio $0.44,95 \% \mathrm{Cl} 0.25-0.78$ ), and Larsen scores were significantly lower in patients with psoriasis who had radiological erosion in the hands and feet (mean 13 vs 28, $P=0.0009$ ).

The authors conclude that patients with inflammatory polyarthritis plus psoriasis have similar 5-year outcomes to patients with RF-negative inflammatory polyarthritis only.

Original article Morgan C et al. (2007) Five-year outcome of a primary-care-based inception cohort of patients with inflammatory polyarthritis plus psoriasis. Rheumatology 46: 1819-1823

\section{IFN- $\alpha$ and BAFF overexpression is associated with etanercept treatment failure in SS}

Treatment has proved difficult for Sjögren's syndrome (SS), a chronic autoimmune disease in which $B$ cells infiltrate and destroy exocrine glands, leading to ocular and oral dryness. Although some pilot studies suggested that treatment with tumor necrosis factor (TNF) antagonists was effective in patients with primary SS, subsequent randomized, controlled trials showed no benefit.

Mavragani et al. hypothesized that treatment of primary SS with etanercept, a potent TNF blocker, might augment an already activated interferon (IFN)- $\alpha$ pathway, raising plasma IFN- $\alpha$ activity and concentrations of BAFF, a TNF family ligand. To investigate this possibility, they used samples from a randomized, double-blind trial to determine plasma IFN- $\alpha$ activity and BAFF levels in 29 healthy controls and in 20 SS patients given either etanercept or placebo twice weekly for 12 weeks. They also used an in vitro system to measure the effect of etanercept on the expression of inducible genes in the IFN- $\alpha$ pathway.

At baseline, SS patients had significantly higher plasma IFN- $\alpha$ activity and BAFF levels than healthy controls. SS patients given etanercept for 12 weeks showed a significant increase in IFN- $\alpha$ activity and BAFF levels compared with those who received placebo. Culturing peripheral blood mononuclear cells in vitro in the presence of etanercept caused a dose-dependent increase in the expression of IFN- $\alpha$ and IFN- $\alpha$-inducible genes.

The authors conclude that their findings could provide a potential explanation for the therapeutic failure of etanercept in SS patients.

Original article Mavragani CP et al. (2007) Augmented interferon-a pathway activation in patients with Sjögren's syndrome treated with etanercept. Arthritis Rheum 56: 3995-4004

\section{Predicting responsiveness to rituximab treatment for rheumatoid arthritis}

Rituximab specifically targets B cells, which have an important role in the pathogenesis of rheumatoid arthritis, effectively depleting concentrations in blood. Information on the effects of this antibody in other tissues is, however, limited. Teng et al. investigated the effects of B-cell depletion in peripheral blood, bone marrow, and synovium 12 weeks after rituximab treatment in 25 patients with severe refractory rheumatoid arthritis. Immunologic characteristics were analyzed to identify 